\title{
Chloroquine enhances the efficacy of cisplatin by suppressing autophagy in human adrenocortical carcinoma treatment
}

This article was published in the following Dove Press journal:

Drug Design, Development and Therapy

7 March 2016

Number of times this article has been viewed

\author{
Liang Qin ${ }^{1, *}$ \\ Tianyuan $\mathrm{Xu}^{1, *}$ \\ Leilei Xia' \\ Xianjin Wang' \\ Xiang Zhang' \\ Xiaohua Zhang' \\ Zhaowei Zhu' \\ Shan Zhong' \\ Chuandong Wang ${ }^{2}$ \\ Zhoujun Shen' \\ 'Department of Urology, Ruijin \\ Hospital, Shanghai Jiao Tong University \\ School of Medicine, ${ }^{2}$ Key Laboratory \\ of Stem Cell Biology, Shanghai \\ Institutes for Biological Sciences, \\ Chinese Academy of Sciences, \\ Shanghai Jiao Tong University School \\ of Medicine, Shanghai, People's \\ Republic of China \\ *These authors contributed equally \\ to this work
}

Background: It has been demonstrated that chloroquine (CQ) enhances the efficacy of chemotherapy. However, little is known about whether CQ could enhance the efficacy of cisplatin (DDP) in the treatment of adrenocortical carcinoma (ACC). In this study, we explore the efficacy and mechanism by which CQ affects DDP sensitivity in human ACC in vitro and in vivo.

Methods: The autophagic gene Beclin-1 expression was detected by immunohistochemistry, and the protein levels were analyzed using immunoblotting assays of ACC tissues and normal adrenal cortex tissues. The ACC SW13 cells were treated with DDP and/or CQ. The cell viability assay was performed using the MTT method. Qualitative autophagy detection was performed by monodansylcadaverine staining of autophagic vacuoles. Annexin V-fluorescein isothiocyanate/propidium iodide double staining was used to count cell apoptosis by flow cytometry. The autophagy-related protein (Beclin-1, LC3, and p62) and apoptosis relative protein (Bax and Bcl-2) levels were evaluated with Western blot analysis. Furthermore, a murine model of nude BALB/c mice bearing SW13 cell xenografts was established to evaluate the efficacy of concomitant therapy.

Results: The expression of the autophagic gene Beclin-1 was significantly downregulated in ACC tissues compared to normal adrenal cortex tissues. The Beclin-1 protein level in ACC tissues was lower than that in normal adrenal cortex tissues $(P<0.05)$. In vitro concomitant therapy (DDP and CQ) was more effective in restraining SW13 cell proliferation. DDP could promote cell apoptosis and induce autophagy in SW13 cells. Concomitant therapy further promoted cell apoptosis by inhibiting autophagy. In vivo, we found that concomitant therapy was more potent than DDP monotherapy in inhibiting the growth of xenografted tumors and prolonging the survival of tumor-bearing mice.

Conclusion: The antitumor ability of DDP was related to autophagy activity, and the concomitant therapy (DDP and CQ) could be an optimal strategy for treating ACC.

Keywords: adrenocortical carcinoma, chloroquine, cisplatin, apoptosis, autophagy

\section{Introduction}

Adrenocortical carcinoma (ACC) is a malignancy of the adrenal cortex; it is rare but very aggressive and has a dismal prognosis. The annual incidence is $0.5-2.0$ cases per one million people, and the median age at diagnosis is 46 years. ${ }^{1,2}$ At the time of diagnosis, approximately $70 \%$ of these malignancies are not limited to the adrenal gland. ${ }^{3}$ With respect to unresectable or widely disseminated ACCs, a number of therapeutic modalities, such as antihormonal drugs, mitotane, systemic chemotherapy, and radiation therapy, can be used to palliate symptoms. However, the 5-year survival of these patients is usually $<15 \% .{ }^{4}$ Cytotoxic therapy (cisplatin, DDP) is an indispensable part of systemic chemotherapy for ACC. ${ }^{5}$ As the most widely tested cytotoxic
Correspondence: Zhoujun Shen Department of Urology, Ruijin Hospital, Shanghai Jiao Tong University School of Medicine, 197, 2nd Ruijin Road, Shanghai 200025, People's Republic of China

$\mathrm{Tel} / \mathrm{fax}+862164315370$

Email shenzj06@sina.com
Drug Design, Development and Therapy 2016:10 1035-1045 Dovepress http://dx.doi.org/10.2147/DDDT.S101701 (c) (1) (5) 2016 Qin et al. This work is published and licensed by Dove Medical Press Limited. The full terms of this license are available at https://www.dovepress.com/terms.php cc) ${ }_{\mathrm{BY}} \mathrm{NC}$ and incorporate the Creative Commons Attribution - Non Commercial (unported, v3.0) License (http://creativecommons.org/licenses/by-n/3.0/). By accessing the work you hereby accept the Terms. Non-commercial uses of the work are permitted without any further permission from Dove Medical Press Limited, provided the work is properly attributed. For permission for commercial use of this work, please see paragraphs 4.2 and 5 of our Terms (https://www.dovepress.com/terms.php). 
drug, DDP has been demonstrated as effective in ACC cell lines. ${ }^{6}$ However, in human, cancers tend to have inherent and acquired resistance to DDP, decreasing its efficacy. ${ }^{7}$ It remains difficult to overcome the drug resistance to DDP in chemotherapy for ACC. Undoubtedly, there are difficulties in treating patients with ACC.

Autophagy, a cellular homeostatic process, involves autophagosomes that sequester the majority of cytoplasmic abnormal or long-lived proteins and organelles; then, the contents are degraded and recycled after they are transported to lysosomes. ${ }^{8}$ Tumorigenesis and tumor development are closely related to autophagy. ${ }^{9}$ Many studies have shown that autophagy can influence the effect of chemotherapy; when chemotherapy causes metabolic deprivation DNA damage, the process of autophagy is activated, resulting in cell survival and resistance to chemotherapy. ${ }^{10,11}$ Additionally, several studies have demonstrated that concomitant therapy with autophagy modulators and chemotherapy drugs can reverse drug tolerance. ${ }^{10,11}$ In mammalian cells, Beclin-1, the autophagic gene, plays a vital role in autophagy. ${ }^{12}$ Growing evidence has shown that aberrant Beclin-1 expression is associated with several tumors, such as hepatocellular carcinoma, colonic carcinomas, and hypopharyngeal carcinoma. ${ }^{13-15}$ However, no studies about the expression of Beclin-1 in ACC have been reported to date.

Chloroquine (CQ), an autophagy inhibitor, has been demonstrated to increase the efficacy of chemotherapy in treating several solid tumors, such as hepatocellular carcinoma, breast cancer, and hypopharyngeal carcinoma. ${ }^{16-18}$ Recent studies on drug resistance mechanisms indicate that the antitumoraugmenting efficacy of CQ is largely due to its autophagyinhibiting effect. ${ }^{19,20}$ In contrast, the chemosensitization and radiosensitization were not enhanced by CQ in small-cell lung cancers or 4T1 tumors. ${ }^{21,22}$ The antitumor-augmenting efficacy of CQ appears to hinge on the context and tumor type. However, little is known about whether CQ could enhance the effects of DDP in treating ACC.

It remains paradoxical whether autophagy is antitumor or tumor-promoting role. According to some reports, autophagy defects could increase tumorigenesis. ${ }^{23,24}$ Meanwhile, other reports have suggested that autophagy can contribute to cancer cell survival in the presence of pressure, and it can even be conducive to tumor metastasis. ${ }^{25,26}$ Previous research has shown that regulating the autophagy level can affect ACC cell proliferation. ${ }^{27}$ In the current study, the protein levels of autophagic gene Beclin-1 were examined in ACC tissues and normal adrenal cortex tissues. Then, the tumor inhibitory efficacy of concomitant therapy (DDP and CQ) in human ACC SW13 cells was compared with DDP monotherapy in vitro and in vivo.

\section{Materials and methods Patients and tissue specimens}

After obtaining study approval from the ethics board of Ruijin Hospital and written informed consent from all patients, we collected the tissue specimens. Thirty-five ACC tissue samples were collected from patients who were diagnosed with ACC and underwent adrenalectomy. An additional 15 tissue samples of normal adrenal glands, which were confirmed by pathologic diagnosis, were collected from patients who were diagnosed with renal carcinoma and underwent radical nephrectomy (including of the ipsilateral adrenal gland). All surgeries were performed in our institution between 2009 and 2014. No chemotherapy or radiotherapy was performed in any of the patients before surgery. The specimens are divided into two parts: one (for immunohistochemistry) was fixed in 10\% formaldehyde solution and the other (for protein extraction) was instantly placed in liquid nitrogen. Two experienced pathologists independently evaluated the samples to determine a diagnosis and perform histological typing. ACC tumor staging was on the basis of the staging system for adrenal tumors set up by the European Network (ENSAT). ${ }^{28}$

\section{Reagents and materials}

CQ and DDP were purchased from Sigma-Aldrich (St Louis, MO, USA). The human ACC SW13 cell line was obtained from the Chinese Academy of Sciences (Shanghai, People's Republic of China). The antibodies used in the experiments included the following: rabbit antibodies against Beclin-1, LC3, Bax, and GAPDH (all from Cell Signaling Technology, Danvers, MA, USA), rabbit antibodies against Bcl-2 (Abcam, Cambridge, UK), and rabbit antibodies against p62 (Proteintech, Chicago, IL, USA). Nude BALB/c mice (4 weeks old, male, 16-20 g) were obtained from the Shanghai Experimental Animal Center, Chinese Academy of Sciences (Shanghai, People's Republic of China).

\section{Immunohistochemical analysis}

For immunohistochemistry, the paraffin-embedded tissue specimens were cut into $4 \mu \mathrm{m}$ thick tissue slides and were then deparaffinized and rehydrated. The specific process was performed as previously described..$^{29}$ Briefly, after antigen retrieval via microwave, the slides were incubated 
with anti-Beclin- 1 antibody $(1: 100)$ overnight at $4^{\circ} \mathrm{C}$. Horse radish peroxidase-conjugated secondary antibody was used to incubate the slides at $37^{\circ} \mathrm{C}$ for 20 minutes. Color development was performed in 3,3'-diaminobenzidine tetrahydrochloride. Under light microscopy, staining was independently evaluated by two experienced pathologists. In terms of the intensity and proportion, Beclin-1 reactivity was evaluated. Beclin-1 reactivity was presented as cytoplasmic and membranous staining that was both granular and diffuse. Four levels (negative, intensity score $=0$; weak, intensity score $=1$; moderate, intensity score $=2$; and strong, intensity score $=3$ ) were used to express the staining intensity. Based on the percentages of stained cells, staining was divided into four categories, including $\leq 25 \%, 26 \%-50 \%, 51 \%-75 \%$, and $>75 \%$ (scored as $0,1,2$, and 3 , respectively). With respect to the sum of the intensity and proportion scores, the index of immunoreactivity was defined as follows: negative, weakly positive, moderately positive, and strongly positive (sum score: 0, 1-2, 3-4, and 5-6, respectively).

\section{Cell line and cell culture}

Roswell Park Memorial Institute (RPMI) 1640 medium was used to culture the cell line supplemented with $10 \%$ fetal bovine serum. Cells were placed in a humid atmosphere of $5 \% \mathrm{CO}_{2}$ and $95 \%$ air at $37^{\circ} \mathrm{C}$ as routine. The media contained glutamine $(2 \mathrm{mM})$, penicillin $(100 \mathrm{IU} / \mathrm{mL})$, and streptomycin $(100 \mu \mathrm{g} / \mathrm{mL})$.

\section{Drug treatment of the cells}

In the experiment, four groups were treated as follows: 1) no drug in the control (Con) group, 2) DDP $(16.7 \mu \mathrm{mol} / \mathrm{L})$ in the DDP group, 3) CQ $(20 \mu \mathrm{mol} / \mathrm{L})$ in the CQ group, and 4) CQ followed by DDP 1 hour later in the combination (DDP + CQ) group. All cells were observed 12, 24, and 48 hours after drug treatment.

\section{Cell viability assay by the MTT method}

The MTT method was used to determine the cell viability as previously described. ${ }^{30}$ Briefly, approximately $5 \times 10^{3}$ SW13 cells were chosen for culture on a 96-well plate overnight. Then, the cells were treated with CQ and/or DDP with the concentration as described earlier. Each group was evaluated in nine duplicate wells at $200 \mu \mathrm{L}$ in volume per well. After drug treatment, MTT $(20 \mu \mathrm{L}, 5 \mathrm{mg} / \mathrm{mL})$ was added to each well. Then, the 96-well plate was incubated for another 6 hours at $37^{\circ} \mathrm{C}$. After the substrate was removed, $150 \mu \mathrm{L}$ of dimethyl sulfoxide was used to dissolve the formazan precipitates in each well. A 96-well microplate reader (Tecan, Swiss) was applied to measure the absorbance value at the $490 \mathrm{~nm}$ wavelength.

\section{Monodansylcadaverine staining for qualitative autophagy detection by fluorescence microscopy}

When cell autophagy is activated, a number of acid autophagic vacuoles appear in the cytoplasm. Cells can absorb monodansylcadaverine (MDC), which is selectively gathered in autophagic vacuole. As a result, MDC can be used as a specific marker for qualitative autophagy detection. ${ }^{31}$ Qualitative autophagy detection was performed by MDC staining of autophagic vacuoles, as previously described. ${ }^{32}$ SW13 cells that were in the logarithmic growth phase were placed in a culture flask. After the cells attached to the wall and the cell growth density was up to $80 \%-90 \%$, the primary culture fluid was discarded, and the cells were washed with phosphatebuffered saline (PBS) solution. Then, each group of cells was treated with drugs as described earlier. MDC solution $(0.05 \mathrm{mmol} / \mathrm{L})$ was added to the cells, which were cultured for 60 minutes and then washed four times by PBS solution. A fluorescence microscope (Olympus BX-60; Olympus Corporation, Tokyo, Japan) was used to observe autophagic vacuoles of SW13 cells. Additionally, MDC-stained autophagic vacuoles were detected using Cell Quest Software (BD Biosciences, San Jose, CA, USA) and then photographed using a fluorescence microscope imaging system.

\section{Annexin V-FITC/PI double staining for apoptosis evaluation by flow cytometry}

SW13 cells at the logarithmic growth phase were placed on a 96-well plate. Each cell group was treated with drugs as previously mentioned. Twenty-four hours after drug treatment, the cells were dissolved by trypsinization and washed with cold PBS for three times. Then, the supernatant fluid was discarded, and binding buffer $(400 \mu \mathrm{L})$ was added. Annexin V-fluorescein isothiocyanate/propidium iodide double staining was performed according to the manufacturer's recommendations (Annexin V-FITC Apoptosis Kit). The excitation wavelength was $488 \mathrm{~nm}$. Annexin V-fluorescein isothiocyanate (FITC) $(6 \mu \mathrm{L})$ and propidium iodide (PI) $(4 \mu \mathrm{L})$ were added to the cells, which were then cultured for 20 minutes. The apoptosis test was conducted in each group using the Cell Quest Software (BD, Franklin Lakes, NJ, USA) in the green and red fluorescence channels. Additionally, flow cytometry (FACScan, BD Biosciences) was used to analyze the results. 


\section{Apoptosis and autophagy-related protein evaluation by Western blot analysis}

For tissue protein extraction, tissues were lysed in radioimmunoprecipitation assay buffer; then, the lysates were centrifuged for 10 minutes at $13,000 \times g$, and the supernatant was retrieved. For cell protein extraction, the cells from each group were harvested and lysed with radio-immunoprecipitation assay $(120 \mu \mathrm{L})$ buffer on ice 24 hours after drug treatment. Immunoblotting assays were conducted as previously described. ${ }^{33}$ In brief, a BCA Assay Kit (Beyotime Biotech, Haimen, People's Republic of China) was used to determine the protein concentrations of the lysates. Afterward, samples were resolved in sample loading buffer (sodium dodecyl sulfate-polyacrylamide gel electrophoresis), heated up to $100^{\circ} \mathrm{C}$ for 5 minutes, and cooled on ice for 5 minutes. Appropriate levels of lysate $(30 \mu \mathrm{g}$ protein/sample) were separated on 'sodium dodecyl sulfatepolyacrylamide gel electrophoresis gel and then transferred to polyvinylidene difluoride (PVDF) membranes. Nonfat milk (5\%) was used to block the PVDF membranes for 1 hour at room temperature. Then, the membranes were incubated at $4^{\circ} \mathrm{C}$ overnight with primary antibodies Beclin-1, LC3, p62, Bax, $\mathrm{Bcl}-2$, and GAPDH that were diluted according to the manufacturer's recommendations. Afterward, the PVDF membranes were completely washed using $0.1 \%$ (V/V) Tris-buffered saline with Tween-20. The membranes were later incubated with the corresponding secondary antibodies (horseradish peroxidase-conjugated; Santa Cruz Biotechnology, Dallas, TX, USA). According to the manufacturer's protocol, we used an electrogenerated chemiluminescence system to visualize the corresponding secondary antibody. ImageJ 1.33 software (National Institutes of Health, Bethesda, MD, USA) was used to quantify the protein bands.

\section{Animal experiments}

After obtaining approval from the Animal Care and Use Committee of Ruijin Hospital, nude BALB/c mice underwent all animal procedures in the nude mouse facility. They were kept in a laminar rack in the specific-pathogen-free environment. SW13 cell suspension $\left(6 \times 10^{6} / 100 \mu \mathrm{L}\right)$ was subcutaneously injected into the left flanks of the mice. When the tumor size increased up to $30 \mathrm{~mm}^{3}$, which was after approximately 18 days, 24 mice were randomly divided into four groups ( $\mathrm{n}=6$ in each group), and drug intervention was started as follows: 1) Con group, treated with $100 \mu \mathrm{L}$ of normal saline; 2) DDP group, treated with $5 \mathrm{mg} / \mathrm{kg}$ DDP in $100 \mu \mathrm{L}$; 3) CQ group, treated with $60 \mathrm{mg} / \mathrm{kg} \mathrm{CQ}$ in $100 \mu \mathrm{L}$; 4) combination group (DDP + CQ), treated with CQ combined with DDP (DDP was given after CQ administration for 20 minutes). All drugs were delivered by intraperitoneal injection. According to the manufacturer's instructions, DDP or CQ was prepared as previously reported. ${ }^{18} \mathrm{CQ}$ was administered daily, and DDP every 6 days. Tumors were measured using a caliper every 4 days, and two perpendicular diameters of each tumor were recorded. The tumor volume was calculated with the following formula: volume $=\left(\right.$ width $^{2} \times$ length $) / 2$.

For survival analysis, the death criteria of the mice were set up as previously reported. ${ }^{20}$ In brief, mice were killed under the following conditions: if the maximal dimension of the tumor exceeded $2 \mathrm{~cm}$, the mouse become moribund, and/or there was skin ulceration caused by tumor growth. We closely monitored the survival state of the mice (four times per day at least). When the mice met any of the death criteria, they were sacrificed; then, the weight and volume of their tumors were measured.

\section{Statistical analysis}

The SPSS software program (version 19.0; IBM Corporation, Armonk, NY, USA) was used for the statistical analyses. Continuous data are expressed as the mean \pm standard deviation. Comparative analyses were performed using the independent sample $t$-test. Categorical data are expressed as the counts (percentages). The relationships between categorical data were analyzed by the chi-square test or Fisher's exact test. The survival curves of mice were drawn using the Kaplan-Meier method and analyzed with the log-rank test. In all tests, a $P$-value $<0.05$ was considered statistically significant.

\section{Results}

\section{Beclin-I is downregulated in ACC tissues}

We analyzed autophagy-related protein Beclin-1 expression by immunohistochemistry in $35 \mathrm{ACC}$ tissues and 15 normal tissues. The positive expression of granular and diffuse Beclin-1 was located on cytomembranes and in cytoplasms of both ACC cells and normal adrenocortical cells (Figure 1A). The results showed that 15 out of 35 (42.9\%) ACC specimens expressed Beclin-1; 12 of these were weakly positive, accounting for $34.3 \%$. However, 14 out of 15 (93.3\%) normal tissues expressed Beclin-1; eight of these were strongly positive, accounting for $53.3 \%$. The positive expression of Beclin-1 in normal tissues was much higher than that in ACC tissues $(P<0.01)$ (Figure 1B).

We also evaluated the associations between clinical pathologic factors of patients with ACC and Beclin-1 expression (Table 1). Negative expression of Beclin-1 was significantly correlated with advanced stage, regional lymph 
A

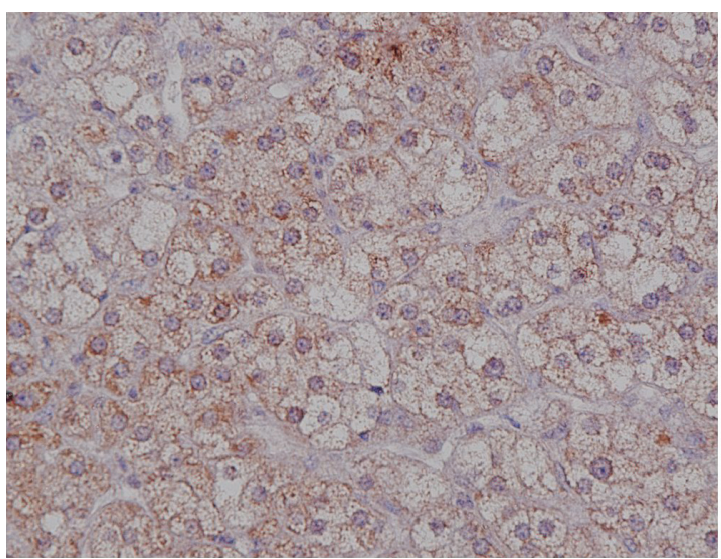

Normal

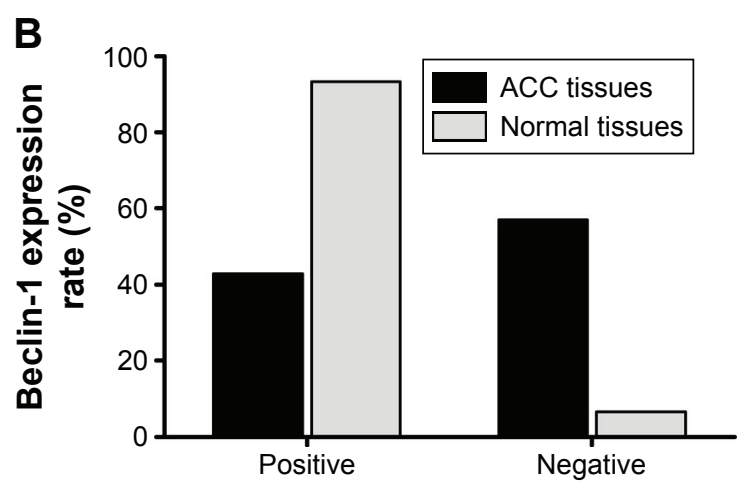

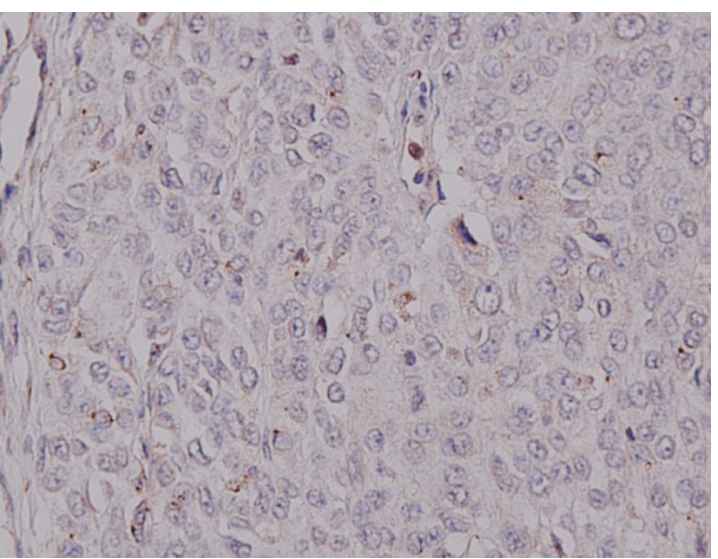

Tumor

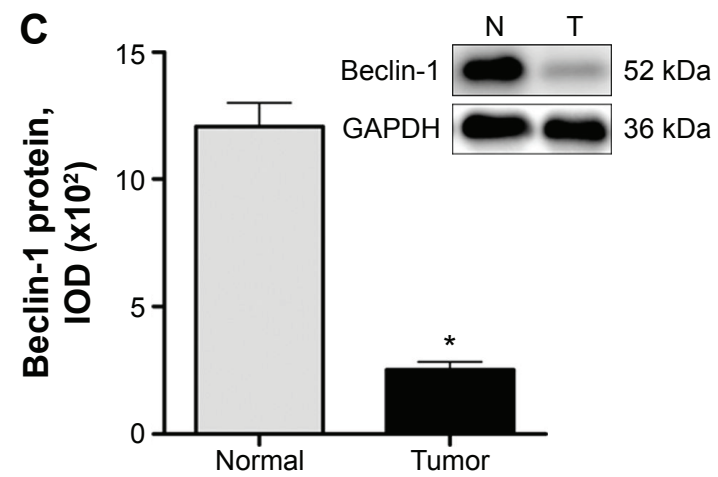

Figure I Beclin-I expression in human ACC and normal adrenocortical tissues.

Notes: (A) Immunohistochemical staining images of normal adrenocortical cells (left) and ACC cells (right). The expression of Beclin-I in normal adrenocortical cells was higher than that in ACC cells (original magnification: $\times 40)$. (B) The expression of Beclin-I was positive in I5 out of 35 (42.9\%) ACC specimens and in I4 out of I5 ( $93.3 \%$ ) normal tissues. (C) Western blot analysis shows that the beclin-I level in ACC tissues was low. Categorical data are expressed as $\mathrm{n}$ (\%). Continuous data are expressed as the mean \pm standard deviation. $* P<0.05$ compared with the normal group.

Abbreviations: ACC, adrenocortical carcinoma; IOD, integrated optical density; $\mathrm{N}$, normal tissue; $\mathrm{T}$, tumor tissue.

node metastasis, increasing $\mathrm{T}$ stage, and poor differentiation ( $P=0.032,0.007,0.019$, and 0.018 , respectively). According to the earlier findings, Beclin-1 expression is significantly downregulated in ACC tissues.

We further detected the Beclin-1 protein expression levels in $15 \mathrm{ACC}$ tissues and 15 normal tissues by immunoblotting assays. The Beclin-1 protein level in ACC tissues was significantly lower than that in normal tissues $(P<0.05)$ (Figure 1C).

\section{Combination of DDP and CQ more efficiently suppresses SWI 3 cell viability}

The effects of CQ and/or DDP on cell proliferation were detected at 12, 24, and 48 hours (Figure 2). Twelve and twenty-four hours after drug treatment, there was no obvious difference in the cell viability among all groups $(P>0.05)$. However, 48 hours after drug treatment, cells in the Con and CQ groups maintained healthy growth, and there was no significant difference in the cell viability between the Con and CQ groups, whereas cells in the DDP and DDP + CQ groups had decreased viability, while the cell proliferation speeds were slower than in the Con group (both $P<0.05$ ). Furthermore, as shown in Figure 2, the cell proliferation speed of the DDP + CQ group is slower than that of the DDP group $(P<0.01)$. The concomitant therapy approach (DDP + CQ) was more efficient at suppressing SW13 cell viability than CQ or DDP monotherapy.

\section{DDP induces autophagy in SWI 3 cells}

Forty-eight hours after drug treatment, there were more MDC-labeled particles of SW13 cells in the DDP group than in the Con group. However, in the DDP + CQ group, a specific autophagy inhibitor (CQ) was added before DDP treatment, and the MDC-labeled particles of SW13 cells obviously decreased (Figure 3A). As a result, DDP can activate cell autophagy, and CQ can reverse this process.

We further analyzed the autophagy-related protein Beclin-1, LC3, and p62 expression levels in SW13 cells by 
Table I Clinicopathologic characteristics of patients with adrenocortical carcinoma and Beclin-I expression

\begin{tabular}{|c|c|c|c|c|}
\hline \multirow[t]{2}{*}{ Characteristics } & \multirow{2}{*}{$\begin{array}{l}\text { Patients, } \\
\text { n (\%) }\end{array}$} & \multicolumn{2}{|c|}{ Beclin-I expression } & \multirow[t]{2}{*}{$P$-value } \\
\hline & & Positive & $\overline{\text { Negative }}$ & \\
\hline Age (range), years & & & & 0.296 \\
\hline$<60(32-59)$ & $21(60.0)$ & II & 10 & \\
\hline$\geq 60(60-74)$ & $14(40.0)$ & 4 & 10 & \\
\hline Sex & & & & 0.596 \\
\hline Female & $19(54.3)$ & 8 & 11 & \\
\hline Male & $16(45.7)$ & 7 & 9 & \\
\hline Clinical stage & & & & 0.032 \\
\hline I-II & $13(37.1)$ & 9 & 4 & \\
\hline III-IV & $22(62.9)$ & 6 & 16 & \\
\hline Tumor stage & & & & 0.019 \\
\hline TI-T2 & $15(42.9)$ & 10 & 5 & \\
\hline T3-T4 & $20(57.1)$ & 5 & 15 & \\
\hline Node metastasis & & & & 0.007 \\
\hline No & $21(60.0)$ & 13 & 8 & \\
\hline $\mathrm{NI}$ & $14(40.4)$ & 2 & 12 & \\
\hline Differentiation & & & & 0.018 \\
\hline Well-moderate & $17(48.6)$ & 11 & 6 & \\
\hline Poor & $18(51.4)$ & 4 & 14 & \\
\hline
\end{tabular}

Notes: Negative expression of Beclin-I was significantly correlated with advanced stage, regional lymph node metastasis, increasing tumor stage, and poor differentiation. Categorical data are expressed as the counts (percentages). The relationships between categorical data were analyzed by the chi-square test or Fisher's exact test.

immunoblotting assays (Figure 3B). Forty-eight hours after drug treatment, the Beclin-1 and $\mathrm{p} 62$ protein expression levels and the LC3-II/I ratio were not obviously different between the DDP $+\mathrm{CQ}$ and Con groups $(P>0.05)$. However, in the DDP group, the Beclin-1 protein expression level and ratio of LC3-II/I were significantly higher than in the Con

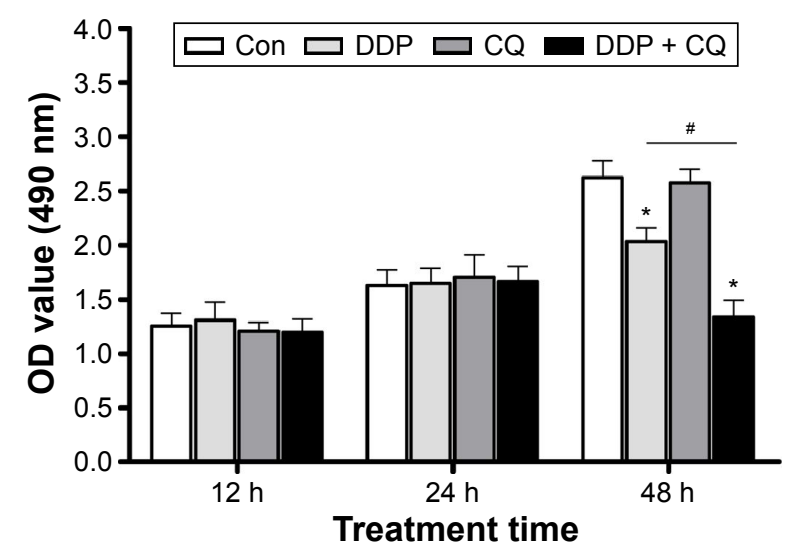

Figure 2 Combination of $\mathrm{CQ}$ and DDP more efficiently suppresses SWI3 cell viability.

Notes: At 12, 24, and 48 hours following drug treatment, cells in the Con and CQ groups maintained healthy growth, whereas cells in the DDP and DDP +CQ groups had decreased viability at 48 hours, which was more notable with the DDP + CQ group. Continuous data are expressed as the mean \pm standard deviation. ${ }^{*} P<0.05$ compared with the control. $\# P<0.01$.

Abbreviations: CQ, chloroquine; DDP, cisplatin; Con, control; OD, optical density; h, hours. group (both $P<0.05$ ); meanwhile, the p62 protein expression level was significantly lower $(P<0.05)$. In the CQ group, the Beclin-1 protein expression level and LC3-II/I ratio were obviously lower than in the Con group (both $P<0.01$ ); also, the p62 protein expression level was obviously higher $(P<0.01)$ (Figure $3 \mathrm{C}$ and $\mathrm{D}$ ).

\section{CQ promotes DDP efficacy to induce SWI 3 cell apoptosis}

Forty-eight hours after drug treatment, there was no obvious difference in the apoptosis rate between the Con and CQ groups $(P>0.05)$, while the apoptosis rates of the Con group were significantly lower than the DDP + CQ and DDP groups (both $P<0.01$ ). Furthermore, the apoptosis rate of the DDP group was significantly lower than the DDP + CQ group $(P<0.01)$ (Figure 4A and $\mathrm{B})$. The concomitant therapy approach (DDP + CQ) was more efficient at inducing SW13 cell apoptosis than DDP monotherapy.

We further detected the apoptosis relative protein Bax and Bcl-2 expression level in SW13 cells by immunoblotting assays. Forty-eight hours after drug treatment, the Bax and $\mathrm{Bcl}-2$ protein expression levels were not significantly different between the Con and CQ groups $(P>0.05)$. The $\mathrm{Bax} / \mathrm{Bcl}-2$ ratio in the DDP group is obviously higher than in the Con group $(P<0.01)$. Furthermore, the $\mathrm{B} / \mathrm{Bcl}-2$ ratio in the DDP + CQ group is higher than in the DDP group $(P<0.01)$ (Figure 4C).

\section{Concomitant therapy approach (DDP + $C Q$ ) increases the efficacy of DDP monotherapy in xenograft mice}

There is no significant difference in the tumor weight or volume between the Con and CQ groups $(P>0.05)$. The tumor volume in the DDP group was significantly lower than in the Con group, and the weight of the DDP group was significantly lighter than the Con group (both $P<0.01$ ). Furthermore, the concomitant therapy approach (DDP + CQ) was more effective in reducing the tumor weight and size compared with DDP monotherapy (both $P<0.01$ ) (Figure 5A and B).

As the Kaplan-Meier curves show there was no obvious difference in survival of the mice in the Con and CQ groups $(P>0.05)$. The survival of the mice increased with DDP monotherapy compared with the Con group, and this effect was much stronger with the concomitant therapy approach (DDP + CQ) (further increased survival of the mice compared with DDP monotherapy) $(P<0.01)$ (Figure 5C). 
A

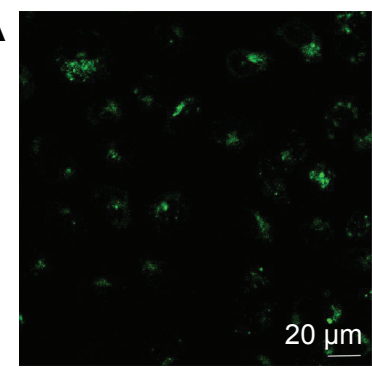

Con

B

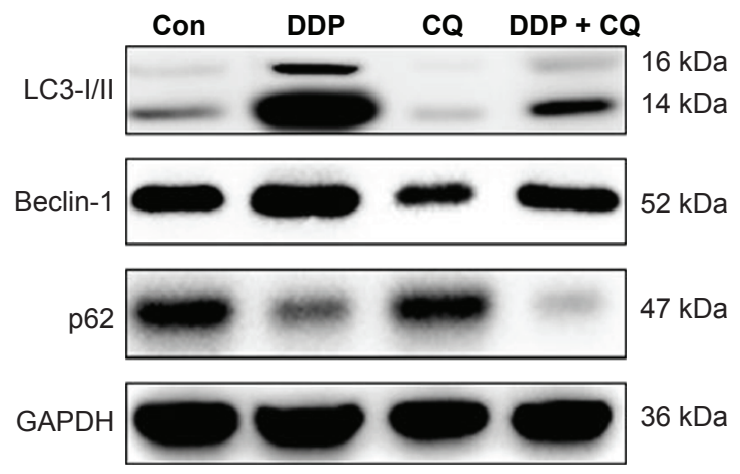

D

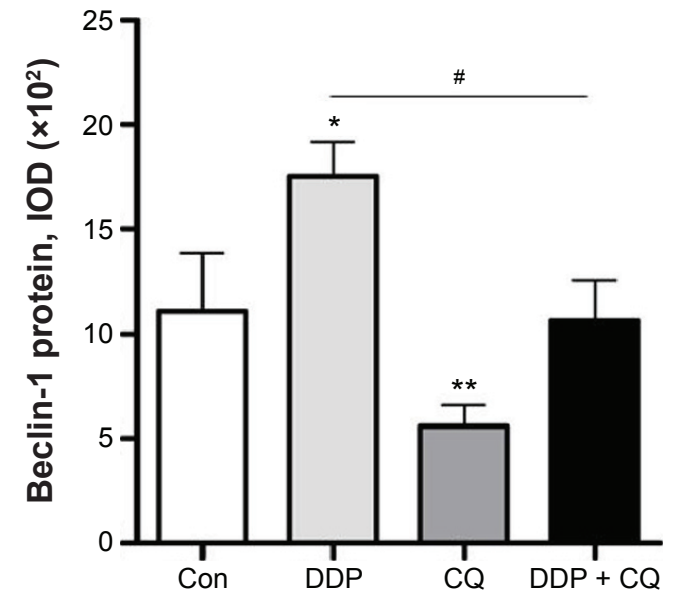

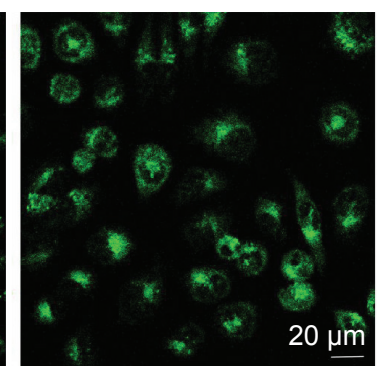

DDP

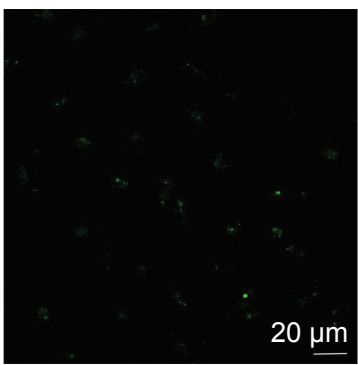

CQ

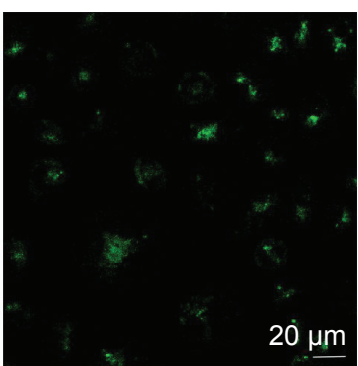

$\mathrm{DDP}+\mathrm{CQ}$
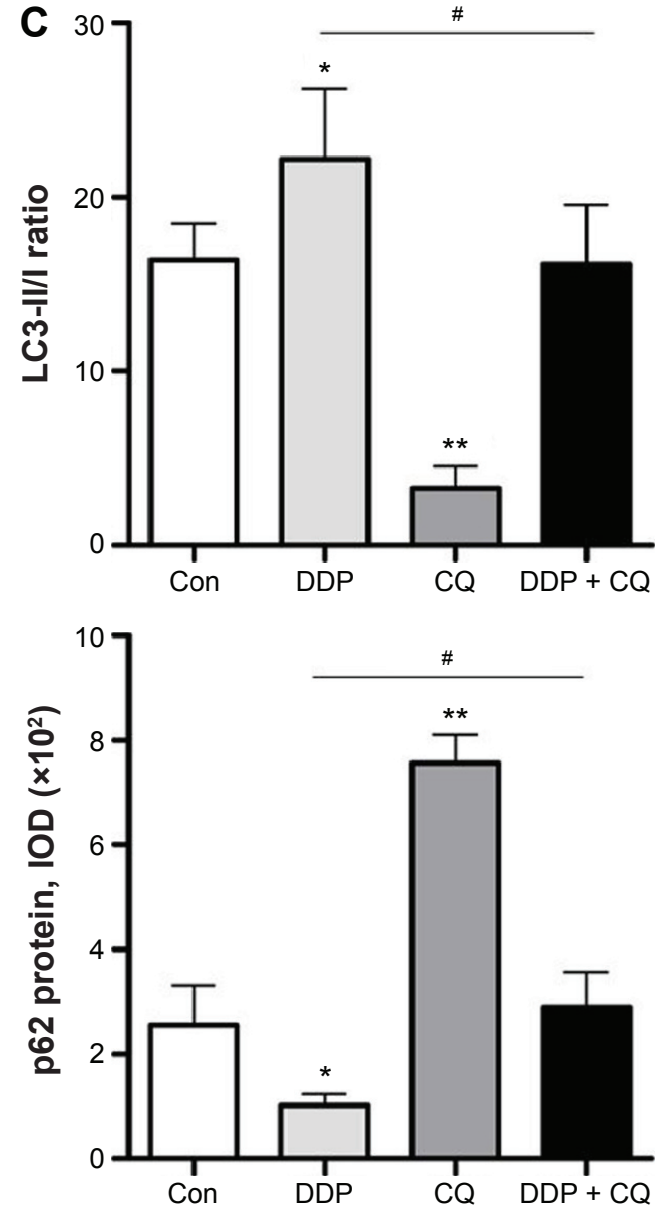

Figure 3 DDP induces autophagy in SWI3 cells Autophagy Vesica.

Notes: (A) Representative MDC staining for observing Autophagy Vesica by fluorescent microscope, scale bars $=20 \mu \mathrm{m}$. The dotted fluorescence structure (MDC staining of autophagic vacuoles) is located around the nucleus. (B) Autophagy-related protein assay (Beclin-I, LC3, and p62) with Western blot analysis. (C) Densitometric analysis of the LC3-II/I ratio. (D) The Beclin-I (left) and p62 (right) expression levels were quantified in terms of the IOD. Continuous data are expressed as the mean \pm standard deviation. ${ }^{*} P<0.05$ compared with the control. ${ }^{*} * P<0.0$ I compared with the control. ${ }^{*} P<0.05$.

Abbreviations: DDP, cisplatin; MDC, monodansylcadaverine; IOD, integrated optical density; Con, control; CQ, chloroquine.

\section{Discussion}

The autophagic gene Beclin-1 is a very important factor in the process of cancer cell differentiation, autophagy, and apoptosis. ${ }^{34}$ The current study is the first to reveal that the autophagic gene Beclin-1 is significantly downregulated in ACC tissues compared with normal adrenal cortex tissues and that negative expression of Beclin-1 is significantly correlated with the clinicopathologic factors of patients with ACC, including the clinical stage, regional lymph node metastasis, pathological $\mathrm{T}$ stage, and differentiation. The Beclin-1 protein, granular and diffuse, was mostly observed in the cytomembranes and cytoplasms of ACC and normal adrenal cortical cells. Moreover, the Beclin-1 protein levels in ACC tissues were lower than in normal adrenal cortex tissues $(P<0.05)$. Thus, we inferred that downregulation of Beclin-1 plays an important role in ACC development. 

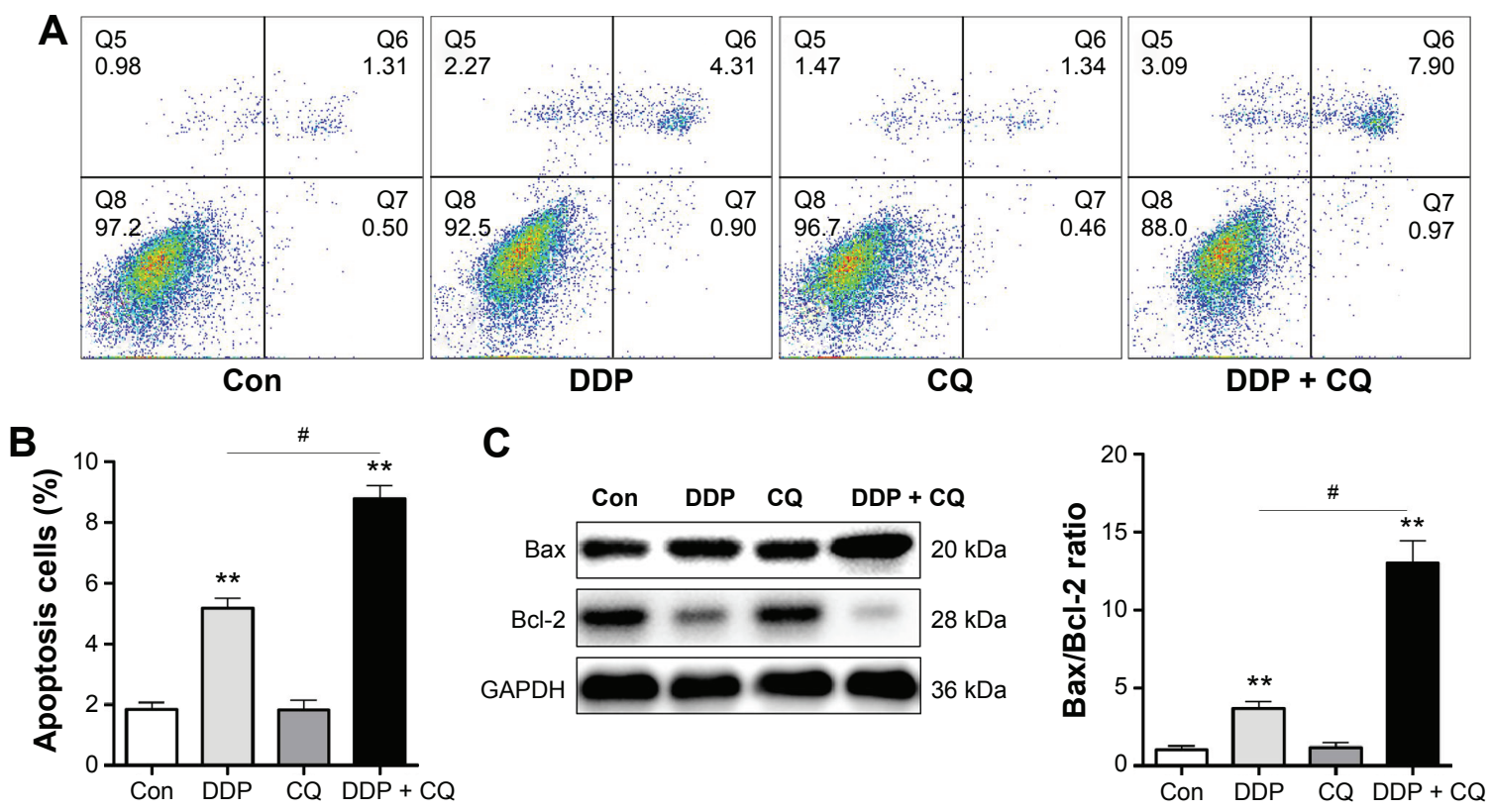

Figure $4 \mathrm{CQ}$ promotes DDP efficacy in inducing SWI3 cell apoptosis.

Notes: (A) Annexin V-FITC/PI was double-stained to detect the apoptosis rate of SWI3 cells. The combination of CQ and DDP is more efficient at inducing SWI 3 cell apoptosis than DDP monotherapy. (B) The data for the apoptosis rate of SWI 3 cells in each group are presented with a bar chart. (C) An apoptosis relative protein assay (Bax and $\mathrm{Bcl}-2$ ) with Western blot analysis (left) and the Bax/Bcl-2 ratio (right) in each group are presented with a bar chart. Continuous data are expressed as the mean \pm standard deviation. $* * P<0.01$ compared with the control. ${ }^{*} P<0.01$.

Abbreviations: CQ, chloroquine; DDP, cisplatin; FITC, fluorescein isothiocyanate; PI, propidium iodide; Con, control.

Christian de Duve first proposed the concept of autophagy and won the Nobel Prize by virtue of this achievement in physiology in $1963 .{ }^{35}$ As a self-digestion procedure in eukaryotic cells, autophagy is type II programmed cell death; ${ }^{36}$ it is in parallel with apoptosis, which has been classified as type I programmed cell death. As part of the cellular homeostatic process, the autophagosome, a double-layered membrane, first wraps abnormal cytoplasmic or long-lived proteins and organelles, allowing for their degradation. They are then recycled after being merged with lysosomes. ${ }^{8}$ Autophagy is characterized by the formation of an autophagic vacuole, which is correlated with the level of the membrane-bound type LC3-II. ${ }^{37}$ The unesterified protein LC3-I can be bound to the $\mathrm{p} 62 /$ sequestosome- 1 protein to degrade ubiquitinated protein, and inhibition of autophagy is associated with the accumulation of 62 protein. ${ }^{38}$ The autophagic flux can also be measured by the LC3-II/I ratio and p62 level.

DDP is an indispensable part of systemic chemotherapy for ACC, ${ }^{5}$ but a major impediment is drug resistance in systemic treatment. ${ }^{7}$ Numerous studies have shown that $\mathrm{CQ}$, an autophagy inhibitor, can reverse drug resistance and further increase the efficacy of chemotherapy and radiotherapy in treating several human cancers. ${ }^{16-18}$ Furthermore, Sotelo et al reported that oral administration of CQ for 12 months ( $150 \mathrm{mg} /$ day), on the basis of the chemotherapy and radiotherapy, prolongs the median survival of patients with glioblastoma compared with placebo in a randomized Phase II clinical trial. ${ }^{39}$ Ren et al reported that DDP-induced apoptosis leads to increased lung cancer cell A549 autophagy, which results in cancer cell survival. ${ }^{40}$

In our experiment, the cell vitality was suppressed after ACC SW13 cells were treated with DDP $(16.7 \mu \mathrm{mol} / \mathrm{L})$ for 48 hours. The cell vitality of the DDP + CQ group is weaker than the DDP group. The concomitant therapy approach (DDP + CQ) was more efficient in suppressing the SW13 cell vitality than DDP monotherapy. Cell autophagy and apoptosis were both increased after ACC SW13 cells were treated with $\operatorname{DDP}(16.7 \mu \mathrm{mol} / \mathrm{L})$, suggesting that DDP-induced apoptosis causes SW13 cell autophagy. In the CQ monotherapy group, there was the lowest number of autophagic vacuoles and autophagy-related protein Beclin-1 expression, while the p62 expression level was the highest, and the LC3-II/I ratio was the lowest in all groups. However, the SW13 cell apoptosis rate and the bax/Bcl-2 ratio were not enhanced compared with the Con group, indicating that CQ $(20 \mu \mathrm{mol} / \mathrm{L})$ can inhibit SW13 cell autophagy viability and has little effect on inhibiting tumor cell growth by itself. After concomitant use of CQ and DDP, the SW13 cell apoptosis rate was significantly increased compared with DDP monotherapy while the cell autophagic activity was at a low level. 

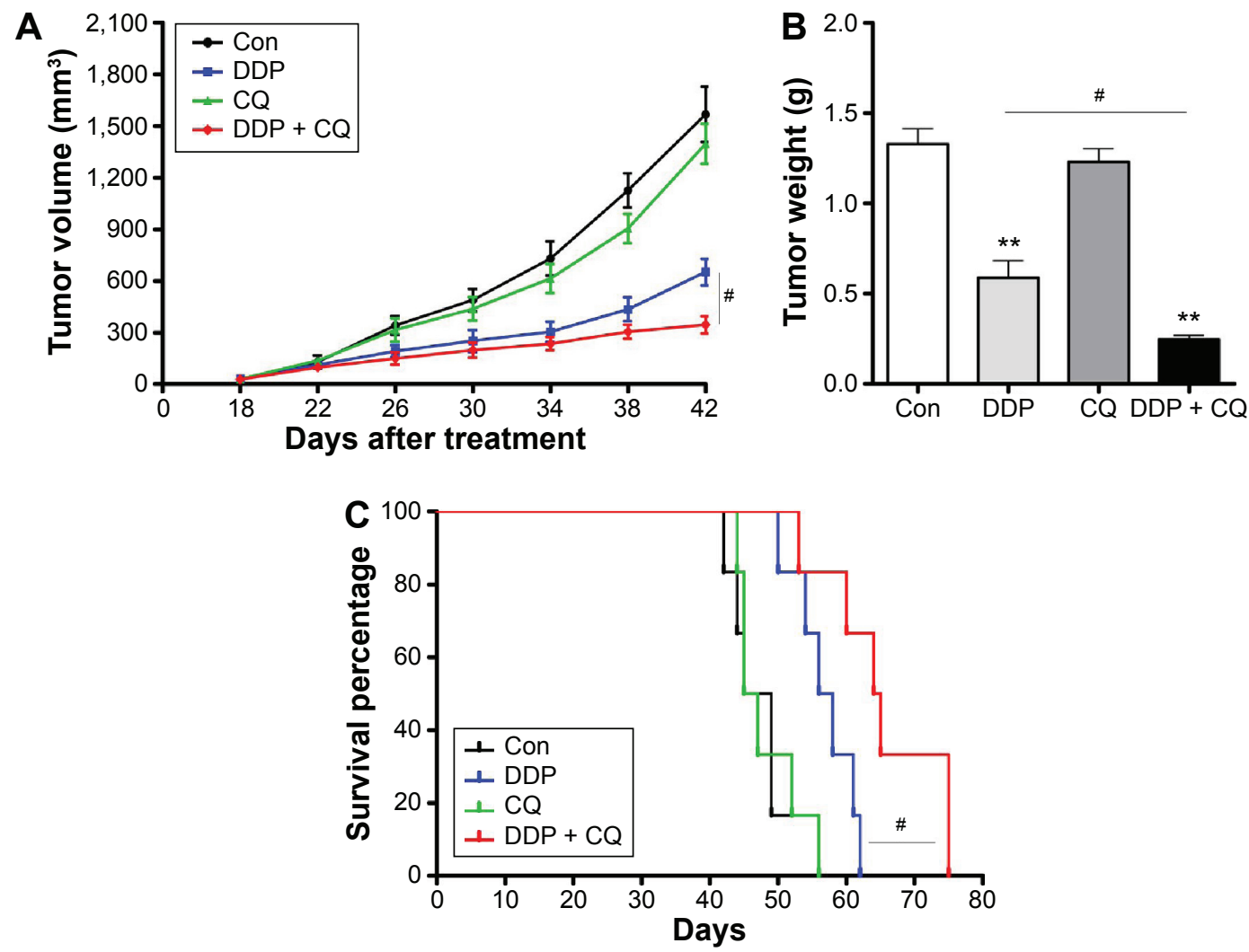

Figure 5 Concomitant therapy approach (DDP + CQ) was more effective than DDP monotherapy in xenograft mice.

Notes: (A) Tumor growth curves of SWI3 xenografts for each group. (B) The tumor weight of SWI3 xenografts for each group. (C) Kaplan-Meier curves for survival analysis of mice. Concomitant therapy approach (CQ + DDP) was much more effective at suppressing tumor growth and prolonging the survival of xenograft mice compared with DDP monotherapy. Continuous data are expressed as the mean \pm standard deviation. $* * P<0.01$ compared with the control. $\# P<0.0$ I.

Abbreviations: DDP, cisplatin; $\mathrm{CQ}$, chloroquine; Con, control.

The aforementioned results indicate that protective autophagy was induced by DDP, and DDP-induced apoptosis of ACC SW13 cells was antagonized by protective autophagy. In other words, protective autophagy results in decreased sensitivity to chemotherapy. The mechanism of the efficacy enhancement of DDP by CQ may be related to inhibition of Beclin-1. In the current study, we also evaluated the efficacy of concomitant therapy approach (DDP and CQ) compared with DDP monotherapy in a murine ACC SW13 tumor model. Our results demonstrated that concomitant therapy with DDP and CQ was more potent than DDP monotherapy in inhibiting the growth of xenografted tumors and prolonging the survival of mice bearing xenografted tumors.

By stabilizing the lysosome membrane and weakening the lysosome acid, CQ, as an autophagy inhibitor, enhances the efficacy of various chemotherapy drugs in treating several human cancers. ${ }^{11,41}$ It is increasingly recognized that CQ facilitates DDP-induced apoptosis. With the addition of $\mathrm{CQ}$, DDP-induced apoptosis was increased, and the efficacy of concomitant drugs was enhanced, but it is not appropriate for all of these advantages to be attributed to inhibiting autophagy. CQ, as a drug with a long history, may enhance the efficacy of chemotherapy by mechanisms other than autophagy. ${ }^{42}$ Maes et al reported that CQ can suppress tumor invasion and metastasis by normalizing tumor vessels to improve the effect of chemotherapy. ${ }^{43}$ Meanwhile, it has also been reported that $\mathrm{CQ}$ can increase the endosomal $\mathrm{pH}$, allowing for sequestration of anticancer drugs via release from the endosome and thereby enhancing the cytotoxic effects of the anticancer drugs. ${ }^{44}$ The detailed mechanisms should be explored in future studies to further evaluate the enhanced efficacy of DDP that is induced by CQ in treating ACC.

\section{Conclusion}

Defective autophagy may play an important role in ACC progression. Autophagy activity was associated with the DDP anticancer effect in in vivo and in vitro experiments. Concomitant therapy with DDP and CQ can increase the efficacy of DDP monotherapy in treating ACC, which may be related to the DDP-induced protective autophagy that was antagonized by CQ. Taken together, a theoretical basis for clinical trials is provided by our research on the concomitant 
use of DDP and CQ for treating. However, it is necessary to verify our findings in more clinical trials.

\section{Acknowledgments}

This project was funded by the National Natural Science Foundation of China (Grant 81472379) and Science and Shanghai Leading Academic Discipline Project (Grant S30201). The authors acknowledge the Shanghai Institutes for Biological Sciences (SIBS) and Chinese Academy of Sciences (CAS), for technical support.

\section{Disclosure}

The authors report no conflicts of interest in this work.

\section{References}

1. Bilimoria KY, Shen WT, Elaraj D, et al. Adrenocortical carcinoma in the United States: treatment utilization and prognostic factors. Cancer. 2008;113:3130-3136.

2. Wang C, Sun Y, Wu H, Zhao D, Chen J. Distinguishing adrenal cortical carcinomas and adenomas: a study of clinicopathological features and biomarkers. Histopathology. 2014;64:567-576.

3. Else T, Kim AC, Sabolch A, et al. Adrenocortical carcinoma. Endocr Rev. 2014;35:282-326.

4. Lughezzani G, Sun M, Perrotte P, et al. The European Network for the Study of Adrenal Tumors staging system is prognostically superior to the international union against cancer-staging system: a North American validation. Eur J Cancer. 2010;46:713-719.

5. Allolio B, Fassnacht M. Clinical review: adrenocortical carcinoma: clinical update. J Clin Endocrinol Metab. 2006;91:2027-2037.

6. Montoya M, Brown JW, Fishman LM. Comparative effects of chemotherapeutic agents on the growth and survival of human adrenal carcinoma cells in culture. Horm Metab Res. 2008;40:302-305.

7. Shen DW, Pouliot LM, Hall MD, Gottesman MM. Cisplatin resistance: a cellular self-defense mechanism resulting from multiple epigenetic and genetic changes. Pharmacol Rev. 2012;64:706-721.

8. Choi AM, Ryter SW, Levine B. Autophagy in human health and disease. N Engl J Med. 2013;368:651-662.

9. Lomonaco SL, Finniss S, Xiang C, et al. The induction of autophagy by gamma-radiation contributes to the radioresistance of glioma stem cells. Int J Cancer. 2009;125:717-722.

10. Chen S, Rehman SK, Zhang W, Wen A, Yao L, Zhang J. Autophagy is a therapeutic target in anticancer drug resistance. Biochim Biophys Acta. 2010;1806:220-229.

11. Levy JM, Thorburn A. Targeting autophagy during cancer therapy to improve clinical outcomes. Pharmacol Ther. 2011;131:130-141.

12. Eskelinen EL, Saftig P. Autophagy: a lysosomal degradation pathway with a central role in health and disease. Biochim Biophys Acta. 2009;1793:664-673.

13. Ding ZB, Shi YH, Zhou J, et al. Association of autophagy defect with a malignant phenotype and poor prognosis of hepatocellular carcinoma. Cancer Res. 2008;68:9167-9175.

14. Li BX, Li CY, Peng RQ, et al. The expression of beclin 1 is associated with favorable prognosis in stage IIIB colon cancers. Autophagy. 2009;5:303-306.

15. Wang J, Pan XL, Ding LJ, Liu DY, Lei D, Jin T. Aberrant expression of Beclin-1 and LC3 correlates with poor prognosis of human hypopharyngeal squamous cell carcinoma. PLoS One. 2013;8:e69038.

16. Shi YH, Ding ZB, Zhou J, et al. Targeting autophagy enhances sorafenib lethality for hepatocellular carcinoma via ER stress-related apoptosis. Autophagy. 2011;7:1159-1172.

17. Cufi S, Vazquez-Martin A, Oliveras-Ferraros C, et al. The antimalarial chloroquine overcomes primary resistance and restores sensitivity to trastuzumab in HER2-positive breast cancer. Sci Rep. 2013; $3: 2469$.
18. Zhao XG, Sun RJ, Yang XY, et al. Chloroquine-enhanced efficacy of cisplatin in the treatment of hypopharyngeal carcinoma in xenograft mice. PLoS One. 2015;10:e0126147.

19. Wu Z, Chang PC, Yang JC, et al. Autophagy blockade sensitizes prostate cancer cells towards Src family kinase inhibitors. Genes Cancer. 2010;1:40-49.

20. Yang S, Wang X, Contino G, et al. Pancreatic cancers require autophagy for tumor growth. Genes Dev. 2011;25:717-729.

21. Zinn RL, Gardner EE, Dobromilskaya I, et al. Combination treatment with ABT-737 and chloroquine in preclinical models of small cell lung cancer. Mol Cancer. 2013;12:16.

22. Bristol ML, Emery SM, Maycotte P, Thorburn A, Chakradeo S, Gewirtz DA. Autophagy inhibition for chemosensitization and radiosensitization in cancer: do the preclinical data support this therapeutic strategy. J Pharmacol Exp Ther. 2013;344:544-552.

23. Gozuacik D, Kimchi A. Autophagy as a cell death and tumor suppressor mechanism. Oncogene. 2004;23:2891-2906.

24. Takamura A, Komatsu M, Hara T, et al. Autophagy-deficient mice develop multiple liver tumors. Genes Dev. 2011;25:795-800.

25. Degenhardt K, Mathew R, Beaudoin B, et al. Autophagy promotes tumor cell survival and restricts necrosis, inflammation, and tumorigenesis. Cancer Cell. 2006;10:51-64.

26. Peng YF, Shi YH, Shen YH, et al. Promoting colonization in metastatic HCC cells by modulation of autophagy. PLoS One. 2013;8:e74407.

27. Cerquetti L, Sampaoli C, Amendola D, et al. Rosiglitazone induces autophagy in H295R and cell cycle deregulation in SW13 adrenocortical cancer cells. Exp Cell Res. 2011;317:1397-1410.

28. Fassnacht M, Johanssen S, Quinkler M, et al. Limited prognostic value of the 2004 International Union Against Cancer staging classification for adrenocortical carcinoma: proposal for a Revised TNM Classification. Cancer. 2009;115:243-250.

29. Zhang X, Wang X, Xu T, Zhong S, Shen Z. Targeting of mTORC2 may have advantages over selective targeting of mTORC1 in the treatment of malignant pheochromocytoma. Tumour Biol. 2015;36:5273-5281.

30. Zhu Z, Xu T, Wang L, et al. MicroRNA-145 directly targets the insulinlike growth factor receptor I in human bladder cancer cells. FEBS Lett. 2014;588:3180-3185.

31. Biederbick A, Kern HF, Elsasser HP. Monodansylcadaverine (MDC) is a specific in vivo marker for autophagic vacuoles. Eur J Cell Biol. 1995;66:3-14.

32. Dai ZJ, Gao J, Ma XB, et al. Antitumor effects of rapamycin in pancreatic cancer cells by inducing apoptosis and autophagy. Int J Mol Sci. 2012;14:273-285.

33. Zhang X, Wang X, Qin L, et al. The dual mTORC1 and mTORC2 inhibitor PP242 shows strong antitumor activity in a pheochromocytoma PC12 cell tumor model. Urology. 2015;85:273.e1-273.e7.

34. Liang XH, Kleeman LK, Jiang HH, et al. Protection against fatal Sindbis virus encephalitis by beclin, a novel Bcl-2-interacting protein. J Virol. 1998;72:8586-8596.

35. Klionsky DJ. Autophagy revisited: a conversation with Christian de Duve. Autophagy. 2008;4:740-743.

36. Levine B, Klionsky DJ. Development by self-digestion: molecular mechanisms and biological functions of autophagy. Dev Cell. 2004;6: 463-477.

37. Kabeya Y, Mizushima N, Ueno T, et al. LC3, a mammalian homologue of yeast Apg8p, is localized in autophagosome membranes after processing. EMBO J. 2000;19:5720-5728.

38. Mathew R, Karp CM, Beaudoin B, et al. Autophagy suppresses tumorigenesis through elimination of p62. Cell. 2009;137:1062-1075.

39. Sotelo J, Briceno E, Lopez-Gonzalez MA. Adding chloroquine to conventional treatment for glioblastoma multiforme: a randomized, double-blind, placebo-controlled trial. Ann Intern Med. 2006;144: 337-343.

40. Ren JH, He WS, Nong L, et al. Acquired cisplatin resistance in human lung adenocarcinoma cells is associated with enhanced autophagy. Cancer Biother Radiopharm. 2010;25:75-80.

41. Kimura T, Takabatake Y, Takahashi A, Isaka Y. Chloroquine in cancer therapy: a double-edged sword of autophagy. Cancer Res. 2013;73:3-7. 
42. Maycotte P, Aryal S, Cummings CT, Thorburn J, Morgan MJ, Thorburn A. Chloroquine sensitizes breast cancer cells to chemotherapy independen of autophagy. Autophagy. 2012;8:200-212.

43. Maes H, Kuchnio A, Peric A, et al. Tumor vessel normalization by chloroquine independent of autophagy. Cancer Cell. 2014;26:190-206.
44. Lee CM, Tannock IF. Inhibition of endosomal sequestration of basic anticancer drugs: influence on cytotoxicity and tissue penetration. $\mathrm{Br}$ J Cancer. 2006;94:863-869.

\section{Publish your work in this journal}

Drug Design, Development and Therapy is an international, peerreviewed open-access journal that spans the spectrum of drug design and development through to clinical applications. Clinical outcomes, patient safety, and programs for the development and effective, safe, and sustained use of medicines are a feature of the journal, which has also been accepted for indexing on PubMed Central. The manuscript management system is completely online and includes a very quick and fair peer-review system, which is all easy to use. Visit http://www.dovepress.com/testimonials.php to read real quotes from published authors.

Submit your manuscript here: http://www.dovepress.com/drug-design-development-and-therapy-journal 